\title{
Finger Probe Array for Topography-Tolerant Scanning Electrochemical Microscopy of Extended Samples
}

\author{
Andreas Lesch, ${ }^{\dagger, \ddagger}{ }^{\dagger}$ Po-Chung Chen, ${ }^{\dagger, \S, \#}$ Folkert Roelfs, ${ }^{\dagger}$ Carsten Dosche, ${ }^{\dagger}$ Dmitry Momotenko, \\ Fernando Cortés-Salazar, ${ }^{\ddagger}$ Hubert H. Girault, ${ }^{\ddagger}$ and Gunther Wittstock ${ }^{*}{ }^{\dagger}$ \\ ${ }^{\dagger}$ Carl von Ossietzky University of Oldenburg, School of Mathematics and Natural Sciences, Center of Interface Science, Department \\ of Chemistry, D-26111 Oldenburg, Germany \\ ${ }^{\ddagger}$ Ecole Polytechnique Fédérale de Lausanne, Laboratoire d’Electrochimie Physique et Analytique, Station 6, CH-1015 Lausanne, \\ Switzerland
}

\section{Supporting Information}

\begin{abstract}
Scanning electrochemical microscopy with soft microelectrode array probes has recently been used to enable reactivity imaging of extended areas and to compensate sample corrugation perpendicular to the scanning direction. Here, the use of a new type of microelectrode arrays is described in which each individual microelectrode can independently compensate corrugations of the sample surface. It consists of conventional Pt microelectrodes enclosed in an insulating glass sheath. The microelectrodes are individually fixed to a new holder system by magnetic forces. The concept was tested using a large 3D sample with heights up to $12 \mu \mathrm{m}$ specially prepared by inkjet printing. The microelectrodes follow the topography in a constant working distance independently from each other while exerting low pressure on the surface.
\end{abstract}

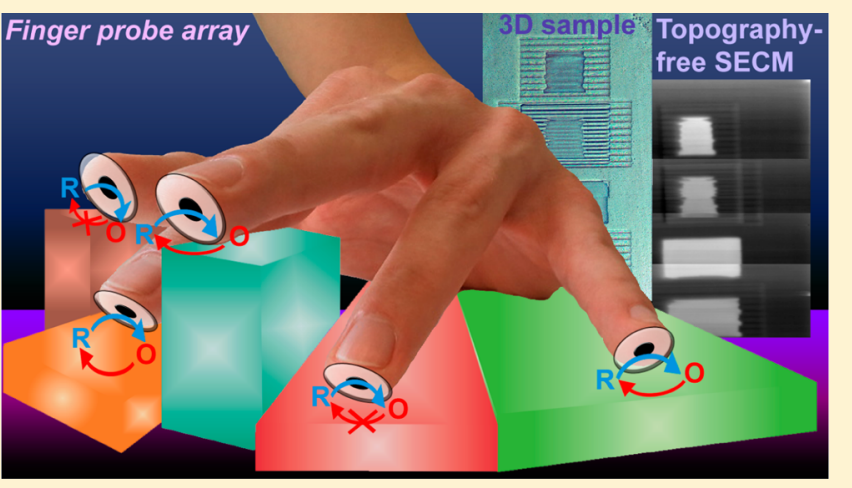

positioning schemes, ${ }^{5-8}$ the application to large image frames for the investigation of catalyst libraries (energy research), ${ }^{9-11}$ latent human fingerprints (forensic science), ${ }^{12-14}$ and testing of technical coatings (corrosion science) $)^{15,16}$ are important for different research directions. ${ }^{17}$

Unfortunately, conventional SECM faces two experimental challenges when working with samples of square centimeter sizes: (i) SECM imaging is time-consuming and (ii) many efforts are required to ensure a constant working distance over the full scan lengths. The first limitation occurs because a finite time $\tau \approx r_{\mathrm{T}}^{2} D^{-1}$ ( $D$ is the diffusion coefficient of the redox mediator) is needed to establish the steady-state diffusion layer. In addition, convective effects may become important when scan rates exceed $10 \mu \mathrm{m} \mathrm{s}^{-1}$ (precisely $v r_{\mathrm{T}} / D \geq 0.06$ over an insulator; $v$ is the horizontal translation rate). ${ }^{18}$ Even when working with scan rates as large as $25 \mu \mathrm{m} \mathrm{s}^{-1}$, it may take $24 \mathrm{~h}$ to record a single centimeter-sized image frame. This is not only time-consuming but enhances the consequences of $\mathrm{ME}$ fouling, substrate aging, solvent evaporation, or instability of reactive species in the electrolyte solution. When being forced to use higher translation rates, SECM image resolution is degraded significantly. The second limitation concerns a change in $d$ during a horizontal line scan either due to the tilt of an

Received: October 2, 2013

Accepted: November 30, 2013

Published: November 30, 2013 
otherwise flat surface or due to the presence of topographic features. Variations in $d$ lead to current changes over a sample of homogeneous reactivity, i.e., those changes are not associated with reactivity differences. Therefore, substantial efforts and skills are required to produce flat substrates and to level them in the SECM setup with respect to the $x, y$-plane of the positioning system using tilt tables and the SECM current as a distance indicator. Recently, Han et al. introduced a substrate leveling method based on an air-bearing rotary stage on which the electrochemical cell with the sample was mounted. ${ }^{19}$ However, for corrugated or $3 \mathrm{D}$ structured substrates even the most sophisticated leveling procedures become useless.

Approaches to overcome these two problems are usually made independently from each other. For instance, in order to investigate or to modify larger areas on a shorter time scale, arrays of MEs have been proposed such as the static $400 \mathrm{ME}$ array introduced by Meyer et al. ${ }^{20}$ The linear microdot array by Barker et al. ${ }^{21}$ consisted of 16 individually addressable Pt MEs, and the $\mathrm{Au}$ multitip-probe by Deiss et al. ${ }^{22}$ were applied for larger areas while keeping the preferred slow probe translation rates. However, these array probes had to be used in constant height mode and require totally flat and aligned surfaces.

Scanning tilted and curved samples or surfaces with significant 3D features becomes very difficult in constant height mode as shown schematically in Scheme $1 \mathrm{a}, \mathrm{b}$ for a

Scheme 1. Comparison of Conventional Constant Height Mode SECM Using Linearly Fixed Arrays of MEs (a,b), Contact Mode SECM with SLMEA (c) and with a FPMEA for Scanning of Samples with Significant Topographic Artifacts (d) ${ }^{a}$
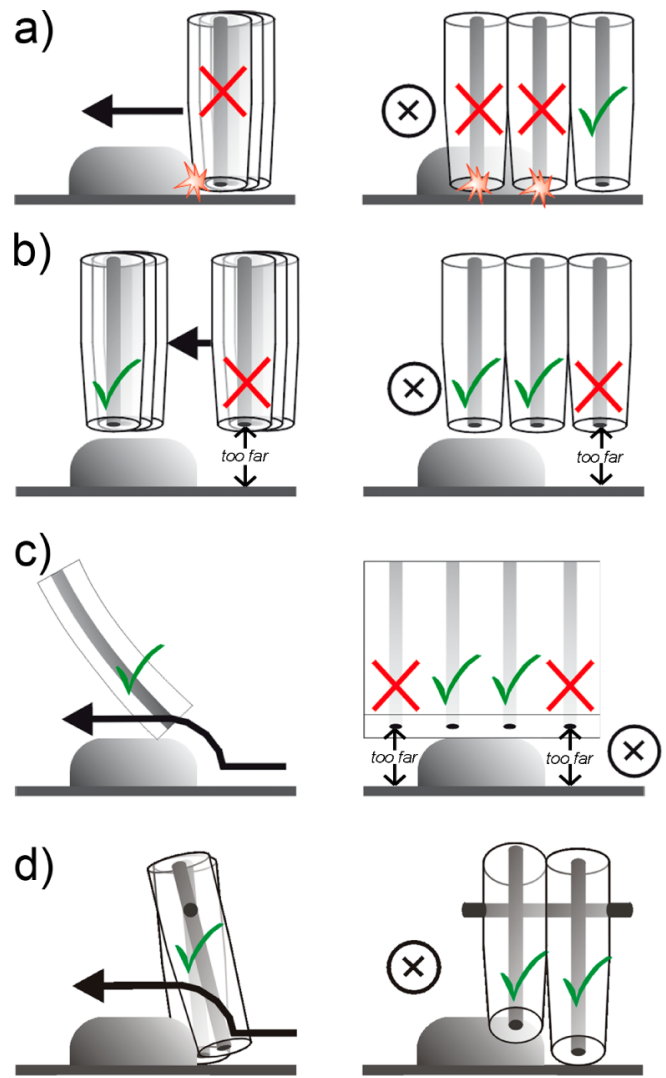

${ }^{a}$ Left column shows the side view on the small side of a probe array; the right column shows side view on the long side of the array. hypothetic linear array of conventional MEs. This schematic applies equally for a linear probe array and an individual probe in the conventional constant height mode. Approaching the ME array to the topographically lowest point of the substrate will cause a ME-sample-crash during a lateral scan if $d$ is smaller than the height of the 3D structure (Scheme 1a). Alternatively, adjusting $d$ close to the highest point of the substrate will avoid a mechanical probe-sample contact, but the SECM will lose the sensitivity for local surface reactivity in the lower laying parts of the surface (Scheme 1b). Four main approaches have been described to overcome the limitations of constant height mode scanning of corrugated substrates, mainly for single probes. One approach is to measure distance-dependent signals that can be directly correlated with $d$, such as the negative feedback current, ${ }^{23,24}$ impedance, ${ }^{25,26}$ or alternating current signals. ${ }^{27}$ In a second approach, SECM is combined with another scanning probe technique which provides the distance control. Well known examples are the combinations of SECM with scanning force microscopy (SFM), ${ }^{28-33}$ electrochemical scanning tunneling microscopy (ECSTM), ${ }^{34}$ and scanning ion conductance microscopy (SICM)., ${ }^{7,3}$ Third, the probe can be vertically vibrated and the reduced damping of this vibration in close proximity to a sample surface can be used to control the probe-substrate distance. Examples are the vertical tip-position modulation (TPM), ${ }^{36,37}$ the detection of shear-forces, ${ }^{5,38,39}$ the hopping intermittent contact mode (HIC), ${ }^{8}$ and the voltage switching mode (VSM). ${ }^{40}$ The fourth approach uses soft SECM probes that are made of flexible polymeric materials that can bend and scan the sample in a brushing-like way thus keeping a constant working distance. ${ }^{41}$ The soft linear microelectrode arrays (SLMEAs) provide a simple and reliable tool for large area investigation and modification. ${ }^{42-45}$ The relatively small pressure that is exerted onto the substrates ${ }^{43}$ even allowed scanning of delicate samples such as selfassembled monolayers without scratching the sample surface or the soft probes. ${ }^{44}$ However, the MEs are embedded in one polymeric block which follows the topographical obstacles as one unit, i.e., all microelectrodes are lifted off if only one electrode encounters an obstacle. Individual sensors will lose the initial close proximity to the surface. The current contrast as well as the resolution will decrease significantly for those electrodes within the array (Scheme 1c). The same effect can be obtained when the sample contains valleys and depressions whose lateral extensions are smaller than the width of the entire soft probe.

Herein, we describe the application of an array of glassshielded Pt MEs capable of independent vertical movements during SECM imaging in contact mode (Scheme 1d). The individual probes of the array climb individually up and down topographic features like fingers exploring the texture of a surface. Therefore, we denoted these probes as finger probe microelectrode array (FPMEA). The advantages are demonstrated using surfaces prepared by using an inkjet printer. The obtained results are compared to SECM images of the same sample using a SLMEA.

\section{EXPERIMENTAL SECTION}

Chemicals. Potassium hexacyanoferrate(II) $\left(\mathrm{K}_{4}\left[\mathrm{Fe}(\mathrm{CN})_{6}\right]\right.$; Merck, Darmstadt, Germany) and potassium dihydrogen phosphate $\left(\mathrm{KH}_{2} \mathrm{PO}_{4}\right.$; Carl Roth, Karlsruhe, Germany) were used as received and were of analytical grade. Deionized water was produced by a Purelab Classic (Elga LabWater, United Kingdom). 
Fabrication of Platinum Microelectrodes. MEs for the FPMEA were prepared by sealing a Pt wire $(25 \mu \mathrm{m}$ in diameter; Goodfellow, Bad Nauheim, Germany) into borosilicate glass capillaries (outer diameter of $1.0 \mathrm{~mm}$, wall thickness of 0.25 $\mathrm{mm}$; Hilgenberg, Malsfeld, Germany). A glass capillary was first vertically pulled into two micropipet tip capillaries by a PC-10 puller (Narishige, Japan) using a heated filament and gravitational force. The resulting tips were then sealed using a Bunsen burner and approximately $1 \mathrm{~cm}$ of Pt wire $(25 \mu \mathrm{m}$ in diameter; Goodfellow, Bad Nauheim, Germany) was inserted into the central of the tip of the capillary. The wire was then sealed into the tip under vacuum using the heated filament of the PC-10 puller. A polished copper wire with suitable length was inserted and glued with silver epoxy at $60{ }^{\circ} \mathrm{C}$ to provide electrical connection to the Pt wire. Afterward, the MEs were mechanically polished and shaped to disk-type electrodes using a Micro Grinder EG-400 (Narishige, Japan). These MEs were inserted into $5.0 \mathrm{~mm}$ long steel tubes $(1.2210115 \mathrm{CrV}, 1.0 \mathrm{~mm}$ inner diameter, $1.4 \mathrm{~mm}$ outer diameter) and fixed using a glue stick (WA11, Pelikan Pritt Klebestift). The disk-shaped MEs were inspected using a reflection mode confocal laser scanning microscope (CLSM, type TCS SP2 AOBS, Leica Microsystems, Wetzlar, Germany). Only MEs with a Pt microdisk centered in the insulating sheath were used to build the FPMEA.

Preparation of 3D Structured Samples. 3D structured samples were prepared on Kapton HN foils (polyimide (PI); $125 \mu \mathrm{m}$ thickness; Goodfellow, Huntingdon, England) by multilayer inkjet printing using the DMP-2831 materials printer (Dimatix Fujifilm, Santa Clara, CA). Jettable nano silver EMD5603 (w/w 20\%) and the UV-curable jettable insulator EMD6201 (both Sun Chemical, Carlstadt, NJ) were used. Three nozzles were simultaneously used for both inks and the printing parameters such as jetting frequency, waveform, voltage, etc. were adjusted for optimum printing performance. The achieved thickness of the 3D patterns was up to $12 \mu \mathrm{m}$ for two layers of the insulator onto a PI substrate. A light guide from an Omnicure S2000 mercury UV lamp (Lumen Dynamics, Mississauga, Ontario, Canada) was mounted to the printhead of the DMP-2831. The Dimatix software allowed opening of the shutter of the UV light guide during printing in order to polymerize the deposited UV curable ink just slightly delayed from the release of the droplets. Silver patterns on PI and on the printed insulator were prepared by the deposition of two layers of the $\mathrm{Ag}$ ink, followed by a curing process ( $30 \mathrm{~min}$ at $200{ }^{\circ} \mathrm{C}$ with heating and a cooling rate of $2{ }^{\circ} \mathrm{C} \mathrm{min}{ }^{-1}$ ). The dimensions and the topography of the patterns were investigated by CLSM in reflection mode and with a DektakXT mechanical profiler (Bruker, Billerica, MA).

SECM Measurements. SECM experiments were carried out using a SECMx system consisting of a Märzhäuser positioning system (Märzhäuser, Wetzlar, Germany), a tilt table (Zaber Technologies, Vancouver, Canada), and a multipotentiostat module MultiWE32 connected to a CompactStat (both Ivium Technologies, Eindhoven, The Netherlands). ${ }^{42,46}$ The multipotentiostat module allowed simultaneous and sequential control of up to 32 working electrodes vs one reference $(\mathrm{RE})$ and one counter electrode $(\mathrm{CE})$. In all experiments, a Pt wire served as $\mathrm{CE}$ and a Ag-wire was used as quasi-RE (QRE) to which all potentials $E_{\mathrm{T}}$ are referred to. Solutions of $4 \mathrm{mM} \mathrm{K}_{4}\left[\mathrm{Fe}(\mathrm{CN})_{6}\right]$ and $0.1 \mathrm{M}$ phosphate buffer $(\mathrm{pH} 7.0)$ were used in all experiments with $E_{\mathrm{T}}=0.3 \mathrm{~V}$. The investigated samples were unbiased.
In all figures, the horizontal scanning direction of SECM line scans and imaging experiments was in the negative $x$-direction, while incremental steps between line scans were performed in the $y$-direction. Herein, the $x$-axis is denoted as the "high frequency" (HF) axis and the $y$-axis is denoted as the "low frequency" (LF) axis due to the frequency of the probe movement. A probe lift-off (LO) was used during reverse scans and during incremental steps in the LF direction within images. This avoids scratching of the glass sheaths of FPMEAs into the substrate.

Data were recorded and plotted online during experiments in the SECMx control software. After the data had been saved, the in-house made software MIRA ${ }^{47}$ was used to process the data using a previously reported calibration routine ${ }^{43,44}$ to correct for slightly varying currents between MEs due to variations in sizes, geometries, and working distances within a FPMEA. In short, the feedback current $i_{\mathrm{T}, k}$ of the individual ME $k$ within an array was calibrated by applying a current offset $i_{\mathrm{T}, k, \text { offs }}$ and a scale factor $s_{k}$ to transform $i_{\mathrm{T}, k}$ into a calibrated, dimensionless current $i_{\mathrm{T}, k}^{\prime} / i_{\mathrm{T}, \max , k}^{\prime}$. The calibration routine sets the current for negative feedback to zero and for positive feedback to unity. Furthermore, the positional offsets of MEs were corrected by applying an $x$-offset $x_{\text {off }, k}$ and a $y$-offset $y_{\text {off }, k}$ for each ME of the array.

\section{RESULTS AND DISCUSSION}

Design of the Finger Probe Array Setup. A schematic representation of the new FPMEA setup is shown in Scheme 2a. A modified version of the previously introduced holder for SLMEAs was used for mounting the new FPMEA. ${ }^{45}$ It can accommodate up to eight MEs separated by $1.5 \mathrm{~mm}$ (four MEs were used in this work). Each ME is individually attached in a curved indentation by magnetic forces (Scheme $2 b$ ) between a $\mathrm{NdFeB}$ permanent magnet embedded in the holder and a steel tube around the ME body (Scheme 2a,b). The assembly stayed in a default position parallel to the magnet surface (Scheme 2b). An external mechanical perturbation, e.g., a mechanical contact between the ME body (Scheme 2c) and the substrate surface, causes a rotary movement of the ME body indicated by the arrow in Scheme $2 \mathrm{~b}$. In contrast to SLMEAs used before, ${ }^{45}$ the bending of the glass shaft of the ME was insignificant under the applied forces. The ME of a FPMEA started to slide on the surface and to rotate slightly in the holder when touching the substrate. The holder design ensured a ME position as close as possible to the magnet and hence to the initial default position and caused the ME body to press slightly against the substrate thus ensuring a permanent probe-sample contact. The holder contained a screw and a rotatable plate (part VI. in Scheme 2a) in order to adjust the inclination angle $\gamma$ manually.

Voltammetric Characterization of the Finger Probe Array. The four MEs of the FPMEA were characterized by cyclic voltammetry $(\mathrm{CV})$ in bulk solution yielding similar sigmoidal responses for the four MEs (Figure 1a). The steadystate currents vary only by $1.5 \%$ from each other attesting to the comparable dimensions of the Pt MEs. This is potentially a great advantage over carbon SLMEAs that showed a larger electrode-to-electrode variability. ${ }^{42}$ The exact radii $r_{\mathrm{T}}$ of the MEs were determined by chronoamperometry (Supporting Information SI-1). A cross-talk between adjacent MEs due to a possible overlap of diffusion layers was negligible since the ME separation $(1500 \mu \mathrm{m})$ is about 120 times larger than the radius of an individual $\mathrm{ME}(\approx 12.5 \mu \mathrm{m})$. Compared to the carbon SLMEAs, the fabrication of the Pt MEs with Pt wires as starting 
Scheme 2. Schematic Representation of New FPMEA Setup ${ }^{a}$

a)
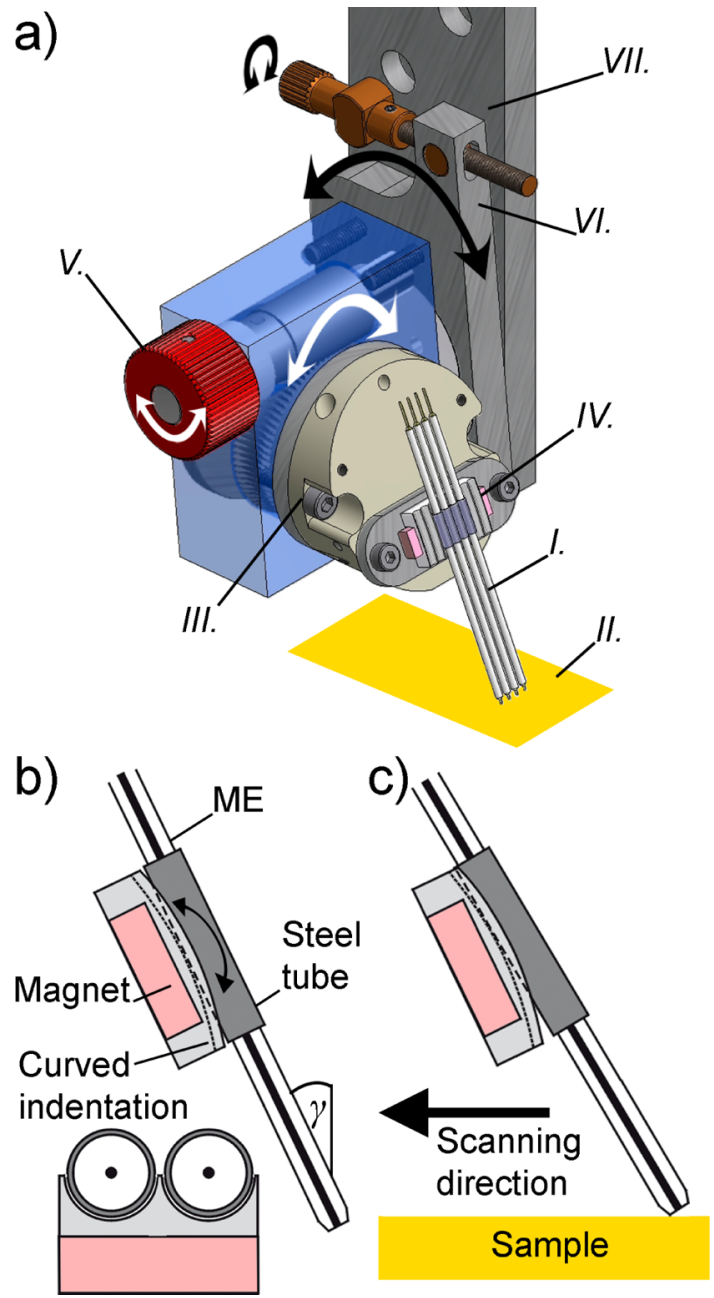

a (a) Scheme of the holder system: I., four individual Pt MEs forming the FPMEA; II., sample; III., holder with preset inclination angle $\gamma$ of $20^{\circ}$ with respect to the surface normal; $I V$., magnet-based holder plate with eight curved indentations for eight MEs; $V$., worm drive for horizontal rotation around an axis in the inclination plane (movements indicated by white arrows); VI., adjustable plate for horizontal rotation around an axis perpendicular to the inclination plane to adjust $\gamma$ (black arrows); VII., plate to mount holder to $x, y, z$-positioning system. (b) Schematic side view of $I V$. in part a when the ME is not in contact with the substrate (initial position). Inset: Cross-section of two MEs in the center position of the holder. (c) Schematic side view of $I V$. in part a when the ME is in contact with the substrate.

material gave slightly better reproducibility with respect to electrode sizes and shapes.

Approach Curves. Experimental SECM feedback mode approach curves using the FPMEA were performed over the insulating PI surface (Figure 1b). The inclination angle $\gamma$ was set to $10^{\circ}$. This angle showed a good current contrast and stable performance during contact mode experiments. Negative feedback behavior, i.e., a decreasing current $i_{\mathrm{T}}$ with decreasing $d$, was obtained due to the hindered diffusion of $\left[\mathrm{Fe}(\mathrm{CN})_{6}\right]^{4-}$ caused by a blocking effect of the sample when the ME is brought in vicinity of the inert and insulating PI substrate. However, the effect of hindered diffusion with $\gamma=10^{\circ}$ is smaller than for perpendicularly mounted MEs. Above a conducting surface, the current at the inclined probe was smaller than for a perpendicularly mounted ME (not shown). The effect of tilted
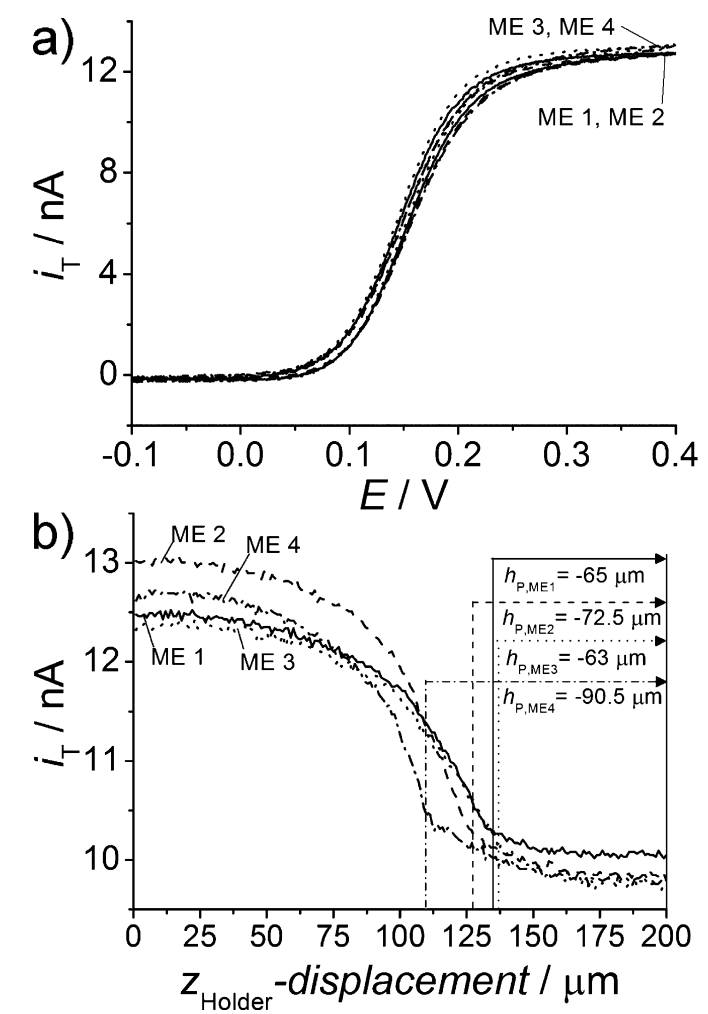

Figure 1. CVs in bulk solution (a) and approach curves toward a polyimide substrate (b) of a FPMEA consisting of four MEs with a lateral separation of $1.5 \mathrm{~mm}$ : (a) scan rate $10 \mathrm{mV} \mathrm{s}^{-1}$. (b) The $z_{\text {Holder }}{ }^{-}$ displacement represents the vertical translation of the holder toward the sample. The vertical lines indicate the $z$-positions where individual MEs made mechanical contact with the PI substrate and started sliding on the substrate surface. Translation rate $1 \mu \mathrm{m} \mathrm{s}^{-1}$.

MEs, i.e., $\gamma \neq 0^{\circ}$, has already been simulated and studied experimentally by Fulian et al., ${ }^{48}$ Sklyar et al., ${ }^{49}$ and Cornut et al. $^{50}$

The measured currents are plotted vs the $z$-displacement of the holder. For each ME, the position of the probe-sample contact is indicated in Figure $1 \mathrm{~b}$ and the $h_{\mathrm{P}}$ value can be identified. The concept of the $h_{\mathrm{p}}$ was described before (Supporting Information SI-2). ${ }^{41-45} h_{\mathrm{P}}$ becomes zero (by definition) upon touching the sample and attains negative values upon further approach of the electrode holder when the probe electrode starts sliding on the sample in the $x$-direction. The vertical position of the probe-sample contact varies between the MEs by $27.5 \mu \mathrm{m}$. This was due to slight variations in the ME shape, their placement in the steel tubes, and the manual mounting procedure of the probes in the holder. Nearly constant currents after mechanical contact indicated almost constant effective working distances $d$ for $h_{\mathrm{P}}<0$. The diffusion hindrance correlated with the glass sheath radius $r_{\text {glass }}$ controlled by the manual production and polishing. These processes are less accurate compared to the automated Parylene $\mathrm{C}$ deposition used for the SLMEAs. The RG values of the MEs are between 8.4 and 11.4 (Supporting Information SI-1) thus showing a variation of $13 \%$. The variations of the current signals between MEs during SECM operations will be caused by variations in active electrodes size $\left(r_{\mathrm{T}}\right)$ and by variation of the effective working distance $\left(r_{\text {glass }}\right)$ (Supporting Information SI-2).

Repetitive approach curves were performed in order to demonstrate that the probes slide on the surface and that the 
vertical position of the MEs in the holder is not affected by the mechanical contact with the sample (Supporting Information SI-3). The $h_{\mathrm{P}}$ value changed between 2.5 to $8.5 \mu \mathrm{m}$ in-between the first and the second approach curves due to a slight upward movement of the MEs in the holder. However, after several approach curves, a steady-state location of the MEs in the holder is achieved and the $h_{\mathrm{P}}$ value of each ME becomes constant for a given $z_{\text {holder }}$-displacement. This allows qualitative SECM imaging (vide infra and Supporting Information SI-3).

The force $F$ which is exerted by a ME of the FPMEA onto the surface in contact mode was measured by approaching a ME toward a PI substrate placed on an analytical balance (Supporting Information SI-4). The obtained spring constant was $k_{\text {Spring }}=-0.64 \mathrm{mN} \mu \mathrm{m}^{-1}$. For a $z_{\text {holder }}$-displacement of $h_{\mathrm{P}}=$ $-20 \mu \mathrm{m}$, this corresponds to an estimated force of $F=12.8$ $\mathrm{mN}$. However, the pressure $p=F / A$ ( $A$ is the contact area) is of higher importance. The contact area of a ME with the substrate is difficult to determine but will most likely be around $26.4 \mu \mathrm{m}^{2}$ as confirmed by optical microscopy of scratches induced by roughly polished MEs. This results in an approximate pressure of $4.9 \times 10^{8} \mathrm{~N} \mathrm{~m}^{-2}$. For comparison, the SLMEA exerted much lower forces $(44-126 \mu \mathrm{N})$ on contact areas of around $1840 \mu \mathrm{m}^{2}\left(p=(2.4-6.9) \times 10^{4} \mathrm{~N}\right.$ $\left.\mathrm{m}^{-2}\right) .{ }^{43}$ Conventional SFM tips in contact mode apply forces in the range of $1 \mathrm{nN}<F<100 \mathrm{nN}$ on a contact area with a diameter of $2-10 \mathrm{~nm}\left(p \geq 1.3 \times 10^{7} \mathrm{~N} \mathrm{~m}^{-2}\right) .{ }^{51}$ Therefore, the pressure that is produced by the ME of the FPMEA exceeds the ones of the SLMEAs and typical SFM tips. Therefore, it is expected that they cause tiny scratches on delicate samples such as self-assembled monolayers.

Horizontal Scans on 3D Structures. Horizontal line scans in contact or contactless feedback mode were performed on the 3D sample (sample design is detailed in Supporting Information SI-5). In contactless mode, i.e., essentially in the conventional constant height SECM mode, the MEs were vertically oriented in the magnetic holder $\left(\gamma=0^{\circ}\right)$ demonstrating that the new holder system can also be used in conventional SECM mode. The working distances were adjusted to similar values as the ones obtained during contact mode scanning, i.e., around $20 \mu \mathrm{m}$ with respect to the PI surface. Please note that this working distance is rather large for a vertically oriented ME but allowed a better comparison of the results obtained in contact mode (see calculated $d$ in the Supporting Information SI-1). Three MEs of the FPMEA were used to scan simultaneously three different characteristic surface structures. The obtained results were plotted in Figure $2 \mathrm{a}-\mathrm{c}$. For a flat insulating surface with a centered conductive $\mathrm{Ag}$ pattern (Figure 2a), the feedback responses recorded in contactless and contact mode showed the same trends. Hindered diffusion was obtained over the insulating part $\left(i_{\mathrm{T}}\right.$ $\left.<i_{\mathrm{T}, \infty}\right)$ and redox mediator regeneration $\left(i_{\mathrm{T}}>i_{\mathrm{T}, \infty}\right)$ over the conducting part. The achieved resolution of both scanning modes was quite similar as indicated by the measured currents and by the shape of the current profiles. As can be seen from the change in the measured currents over the insulator in contactless mode, the sample was slightly tilted which affected the working distance. The FPMEA followed this tilt in contact mode and kept a constant working distance resulting in an essential flat signal over the PI substrate. This advantage of the FPMEA concept becomes more apparent in Figure $2 b$ where a completely insulating surface with a height of about $4 \mu \mathrm{m}$ was scanned (height profiles of the sample in the Supporting Information SI-5). In contactless mode, $d$ and hence the
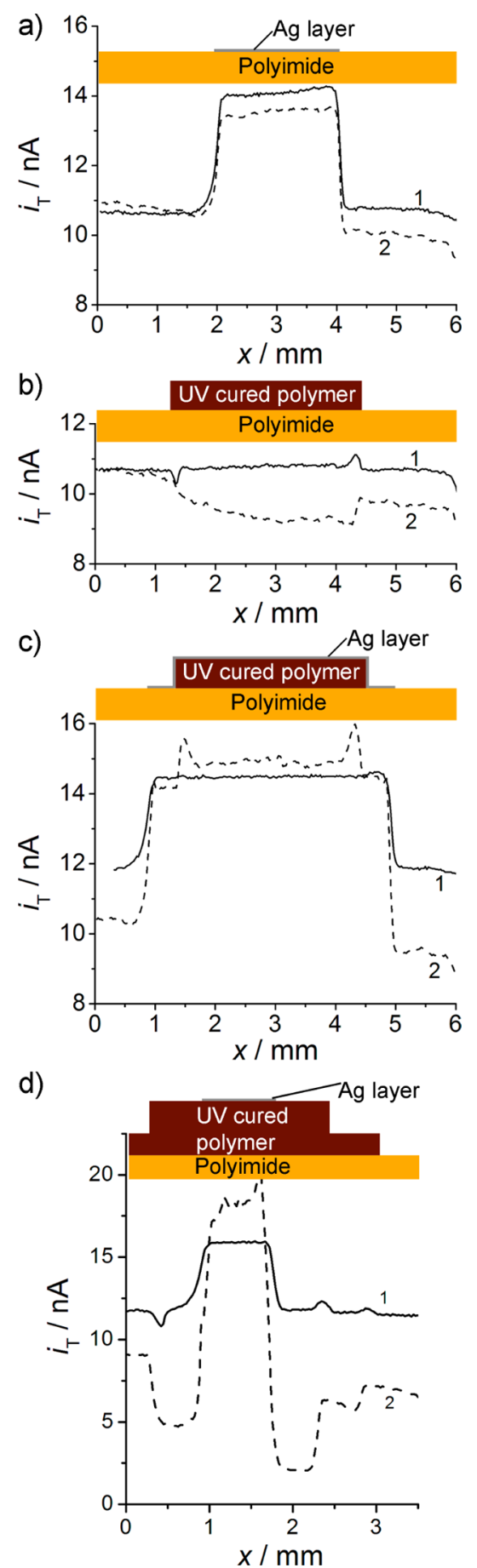

Figure 2. Comparison of line scans in contact $\left(1, \gamma=10^{\circ}\right)$ and contactless $\left(2, \gamma=0^{\circ}\right)$ SECM feedback mode using the FPMEA. The scanned substrate regions were flat with a Ag pattern in the middle (a), insulating with a topographic step (b), a step covered with $\mathrm{Ag}$ (c), and two steps of printed insulator with a centered $\mathrm{Ag}$ pattern (d). Experimental conditions: translation rate $=25 \mu \mathrm{m} \mathrm{s} \mathrm{s}^{-1}, h_{\mathrm{p}}$ values (contact mode) $=-34 \mu \mathrm{m}(\mathrm{a}),-26 \mu \mathrm{m}(\mathrm{b}),-38 \mu \mathrm{m}(\mathrm{c})$, and -30 $\mu \mathrm{m}(\mathrm{d}) ; d$ (contactless mode) $=20 \mu \mathrm{m}$ with respect to the PI substrate $(\mathrm{a}-\mathrm{c})$ and $15 \mu \mathrm{m}(\mathrm{d})$.

current response changed due to the tilt of the sample and the height of the polymer block on the substrate. In contrast, the $\mathrm{ME}$ followed the topography in contact mode and an almost constant current was obtained. A slight increase of the current was obtained when the ME was slid on top of the UV-cured 
polymer. This can be explained by a change of $\gamma$ and $\alpha$ which represents the angle between the sample surface and the probe surface including the Pt microdisk (Supporting Information SI2 ). An increase of $\alpha$ also augmented $d$. In Figure 2b, two small spikes in the measured current profile were found when the ME passes the edges of the 3D pattern. Because of the large $r_{\text {glass }} d$ temporarily increased when the probe climbed up and decreased when the probe climbed down. These observations were confirmed on a sample entirely coated with $\mathrm{Ag}$ (including the protruding UV-cured polymer, Figure $2 \mathrm{c}$ ). Figure $2 \mathrm{~d}$ shows line scans of a 3D structure consisting of two stairs of 4 and 12 $\mu \mathrm{m}$ heights on the PI substrate using one ME of the FPMEA. A Ag pattern was deposited in the center of the highest plateau. The scan in contactless mode showed clearly the double decrease of the negative feedback current due the two stairs of insulator besides a clear current increase above the Ag layer due to mediator regeneration while contact mode line scanning gave the real surface reactivity.

The contact mode line scans resulted in clear current profiles that represent the real surface reactivity of the substrate while line scans in constant height mode cannot easily distinguish between reactivity or topography changes. Heights up to $12 \mu \mathrm{m}$ were tolerated in contact mode indicated by the nearly constant currents above the insulating areas of the model sample. Only minor variations related to the change in $\alpha$ and the edge-effect can be seen.

SECM Imaging of Large and Complex 3D Samples. High-throughput feedback mode imaging of the 3D structure (Figure 3a) was performed using the FPMEA consisting of four

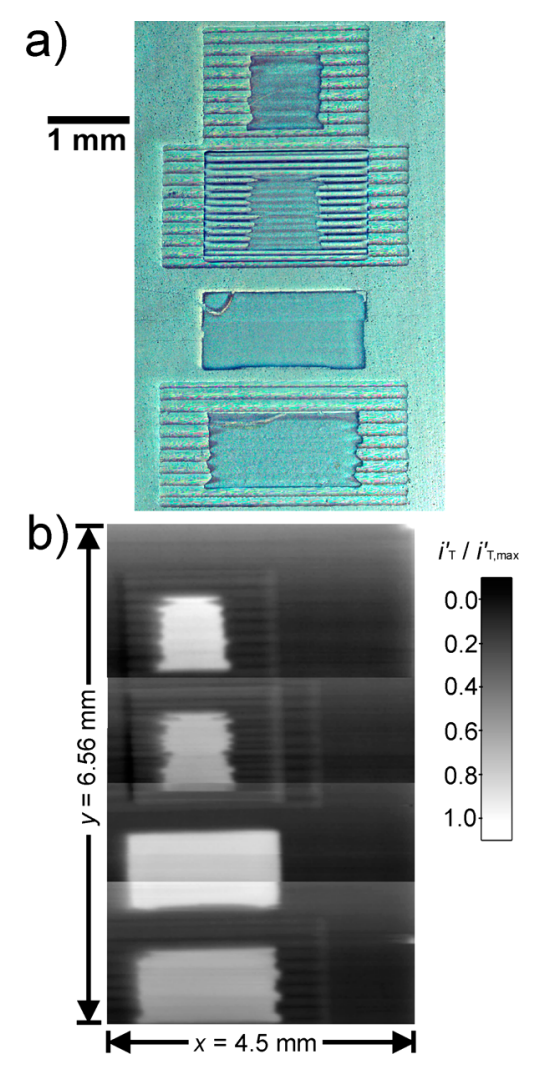

Figure 3. SECM feedback mode imaging of the 3D sample: (a) CLSM image of the scanned pattern after the experiments, (b) SECM feedback image. Detailed imaging parameters are given in the Supporting Information SI-7.
MEs. For comparison, an image using a SLMEA is shown in the Supporting Information SI-6 and demonstrated the entire probe is lifted. The FPMEA was approached to $h_{\mathrm{P}}$ values of $-(23-50.5) \mu \mathrm{m}$ on the PI substrate ensuring a probe-sample contact during imaging. Each of the four MEs scanned simultaneously a sample area of $4.5 \mathrm{~mm}$ ( $x$-direction) by 2 $\mathrm{mm}$ ( $y$-direction) in feedback mode in only $10 \mathrm{~h} 35 \mathrm{~min}$ with a point density of $1629 \mathrm{~mm}^{-2}$ excluding double scanned areas (Supporting Information SI-7). Slower reverse translation rates were selected than for the SLMEA $\left(25 \mu \mathrm{m} \mathrm{s}^{-1}\right.$ instead of 1000 $\mu \mathrm{m} \mathrm{s}^{-1}$ ) in order to prevent displacements of the magnetically attached MEs in the curved indentations of the electrode holder. The horizontal separation of the indentations in the ME holder is $1.5 \mathrm{~mm}$. However, the real separation of the individual MEs in the $y$-direction showed a slight variability due to uncertainties in the exact fitting between glass tubes and steel tubes as well as between steel tubes and grooves of the electrode holder. Therefore, an additional scan length in $y$ direction of $0.5 \mathrm{~mm}$ was used in order to ensure complete coverage of the investigated sample area. The currents measured for the hindered diffusion were equal on the PI and on the UV-cured polymer. Since the $r_{\text {glass }}$ values (here between 108.4 and $136.4 \mu \mathrm{m}$; Supporting Information SI-1) were larger than the width of the ditches in the UV-cured polymer (cross section in Supporting Information SI-5), the MEs cannot follow completely the topography inside these grooves. Because of the slightly enhanced $d$ above the grooves of the printed polymer structure, the inkjet-printed lines appear as slightly enhanced currents in the SECM image. Redox mediator regeneration was obtained over all $\mathrm{Ag}$ patterns, regardless of whether they were printed on PI or on the two layers of the UV-cured polymer. In addition to the results obtained during line scans in contact mode (vide supra), these feedback images did not only confirm the capabilities of topography-tolerant contact mode imaging of heights of up to $12 \mu \mathrm{m}$, they also demonstrate that the MEs were appropriately attached and remained in their holder position while having the possibility to move up and down when encountering a topographic obstacle on the sample or during lift-off in the reverse line scans. The accumulated vertical and horizontal displacement of each individual ME in Figure 3 amounted to 77 $\mathrm{cm}$, underlining the stability of the ME attachment and illustrating the prospective advantages of this new FPMEA. Figure $3 \mathrm{~b}$ shows the SECM image corrected for positional offsets, current offsets, and scale factors (values in the Supporting Information SI-7). Please note that the transformation of the currents of the FPMEAs is a calibration and not a normalization (Supporting Information SI-8). It is currently intended and limited to make high-throughput contact mode imaging of large surface areas. Like the SLMEA, they are currently not suitable for extracting quantitative kinetic data since knowledge of exact working distances, electrode shapes, and insulating sheaths of the individual MEs in a FPMEA would be essential. For each new FPMEA experiment, i.e., after polishing the MEs or when applying a new batch of MEs, the calibration routine has to be repeated. However, this procedure is not very time-consuming using the software MIRA. The current offsets and scale factors for the individual MEs show much less variability than the factors for the carbon SLMEA demonstrating that the Pt MEs are more similar to each other with respect to electrode size and achieved working distance. Positional offsets were corrected by adjusting the surface features of the sample 
pattern. For areas, that were imaged twice, the first images were used. When imaging the same sample region the second time (in the overlap area of two image frames), less homogeneous redox mediator regeneration was observed possibly due to $\mathrm{Ag}$ oxidation in the aqueous solution or lost adhesion between the inkjet-printed and cured Ag pattern during long-term use in the electrolyte solution (Supporting Information SI-7). In the overlap area of the two image frames, damages made by the mechanical contact between FPMEA and Ag pattern in the first image frame could also be detected in the second image frame. However, the CLSM image, taken after the experiment (Figure $3 a)$, showed that these damages are not severe. Because of their different vertical positions in the electrode holder, the individual MEs exerted different pressures on the sample. In addition, small differences in the manual $\mathrm{ME}$ polishing procedures caused variations in the edge definition of the outer rim of the glass sheath leading to slightly variable contact areas and hence variable pressure on the sample. However, the corrected SECM image (Figure 3b) showed a clear and defectfree surface reactivity image that can be directly correlated to the CLSM image of the sample (Figure 3a) but was remarkably free of topographic artifacts that on the other hand would be expected from a constant height image. It should also be noted that a shear force imaging of the same structure would require a prohibitive long time during which the system is unlikely to allow sufficiently constant conditions for the detection of shear forces. $^{52}$

\section{CONCLUSION}

The new concept of a topography-tolerant FPMEA for SECM imaging in contact mode represents a simple, reliable, and fast tool for topography-tolerant contact mode SECM imaging of relatively large samples with $3 \mathrm{D}$ structures. The new probe array is based on conventional Pt MEs and is thus an attractive add-on for all conventional SECM instruments. The probes are placed in curved indentations and are held by magnetic forces. This enables contact mode scanning with a constant working distance due to the individual finger-like movement of these MEs. Large and complex 3D samples can be investigated and highly resolved reactivity images are recorded free of significant topographic artifacts. The system can also be applied with a single ME and on a smaller scale.

Compared to previously introduced carbon ${ }^{41}$ and gold ${ }^{43}$ SLMEA, Pt can be used as electrode material in FPMEAs offering an expanded variety of electrochemically detectable compounds, e.g., $\mathrm{O}_{2}$ or $\mathrm{H}_{2} \mathrm{O}_{2}$, and due to the glass sheath it is also compatible with organic solvents.

Both the SLMEA and the FPMEA can be applied in the contact regime for a broad range of samples including tilted and curved large substrates. The pressure exerted by the SLMEA is significantly smaller enabling its use even for delicate samples such as self-assembled monolayers. The FPMEA will damage such delicate samples but will better resist abrasion when brushing on hard materials. Coating the glass sheath of the MEs with thin polymer films could reduce the risk of mechanical sample damage. The SLMEA can compensate topographic features if these features are extended and perpendicular to the HF scan direction. In contrast, the FPMEA can work on samples with topographic features in arbitrary orientations without additional distance control instrumentation.

As a possible enhancement, laser technology could sense the movement of the individual MEs in the holder and provide simultaneous information about the vertical dimensions of the sample under study. A smaller working distance and thus a higher resolution would be achieved by using MEs with smaller RG values. The number of MEs can be enlarged without fundamental difficulties making it possible to work on large samples such as industrial metallic components of square centimeter sizes or larger, for instance, to test the integrity of corrosion-protective coatings. In this way, SECM is on the way to achieve reactivity imaging from the nanometer up to the macroscale.

\section{ASSOCIATED CONTENT}

\section{Supporting Information}

Tables of the MEs dimensions, the definition of the $h_{\mathrm{P}}$ value, demonstration experiments of the stability of the ME positions in the new probe holder, measurements of the force between MEs and sample, details about the $3 \mathrm{D}$ sample design and topography, a comparative SECM feedback images of the 3D structure using a SLMEA and calibration values, and experimental details for the FPMEA SECM image. This material is available free of charge via the Internet at http:// pubs.acs.org.

\section{AUTHOR INFORMATION}

\section{Corresponding Author}

*E-mail: gunther.wittstock@uni-oldenburg.de. Phone: +49 441 798 3971. Fax: +49 4417983979.

\section{Present Address}

${ }^{\S}$ P.-C.C.: Institute of Systems Biology and Bioinformatics, National Central University, No. 300, Jhongda Rd., Jhongli City, Taoyuan County 32001, Taiwan (R.O.C.).

\section{Author Contributions}

The manuscript was written through contributions of all authors. All authors have given approval to the final version of the manuscript.

\section{Author Contributions}

${ }^{\#}$ A.L. and P.-C.C. contributed equally to this work.

Notes

The authors declare no competing financial interest.

\section{ACKNOWLEDGMENTS}

The work was jointly supported by the Deutsche Forschungsgemeinschaft (DFG, Wi 1617/10) and the Fonds National Suisse pour la Recherche Scientifique (FNSRS, Grant No. 20PA21 121570/1) under the title "High throughput SECM imaging”. P.-C.C. thanks the National Science Council of Tawain for his research grant (Grant No. 101-2917-I-564-033). The technical assistance by Valérie Devaud and Cyrille Hibert (both Ecole Polytechnique Fédérale de Lausanne (EPFL)) is acknowledged.

\section{REFERENCES}

(1) Wittstock, G.; Burchardt, M.; Pust, S. E.; Shen, Y.; Zhao, C. Angew. Chem., Int. Ed. 2007, 46, 1584-1617.

(2) Sun, P.; Laforge, F. O.; Mirkin, M. V. Phys. Chem. Chem. Phys. 2007, 9, 802-823.

(3) Bard, A. J., Mirkin, M. V., Eds. Scanning Electrochemical Microscopy, 2nd ed.; CRC Press: Boca Raton, FL, 2012.

(4) Kwak, J.; Bard, A. J. Anal. Chem. 1989, 61, 1221-1227.

(5) Ballesteros Katemann, B.; Schulte, A.; Schuhmann, W. Chem.Eur. J. 2003, 9, 2025-2033.

(6) Sun, P.; Laforge, F. O.; Abeyweera, T. P.; Rotenberg, S. A.; Carpino, J.; Mirkin, M. V. Proc. Natl. Acad. Sci. U.S.A. 2008, 105, 443448. 
(7) Takahashi, Y.; Shevchuk, A. I.; Novak, P.; Murakami, Y.; Shiku, H.; Korchev, Y. E.; Matsue, T. J. Am. Chem. Soc. 2010, 132, 1011810126.

(8) Lazenby, R. A.; McKelvey, K.; Unwin, P. R. Anal. Chem. 2013, 85, 2937-2944.

(9) Fernandez, J. L.; Walsh, D. A.; Bard, A. J. J. Am. Chem. Soc. 2005, 127, 357-365.

(10) Minguzzi, A.; Alpuche-Aviles, M. A.; López, J. R.; Rondinini, S.;

Bard, A. J. Anal. Chem. 2008, 80, 4055-4064.

(11) Wain, A. J. Electrochim. Acta 2013, 92, 383-391.

(12) Zhang, M.; Girault, H. H. Electrochem. Commun. 2007, 9, 17781782

(13) Cortes-Salazar, F.; Busnel, J.-M.; Li, F.; Girault, H. H. J. Electroanal. Chem. 2009, 635, 69-74.

(14) Zhang, M.; Qin, G.; Zuo, Y.; Zhang, T.; Zhang, Y.; Su, L.; Qiu, H.; Zhang, X. Electrochim. Acta 2012, 78, 412-416.

(15) Pilbáth, A.; Szabó, T.; Telegdi, J.; Nyikos, L. Prog. Org. Coat. 2012, 75, 480-485.

(16) Souto, R. M.; Gonzalez-Garcia, Y.; Izquierdo, J.; Gonzalez, S. Corros. Sci. 2010, 52, 748-753.

(17) Cortes-Salazar, F.; Momotenko, D.; Girault, H. H.; Lesch, A.; Wittstock, G. Anal. Chem. 2011, 83, 1493-1499.

(18) Combellas, C.; Fermigier, M.; Fuchs, A.; Kanoufi, F. Anal. Chem. 2005, 77, 7966-7975.

(19) Han, L.; Yuan, Y.; Zhang, J.; Zhao, X.; Cao, Y.; Hu, Z.; Yan, Y.; Dong, S.; Tian, Z. Q.; Tian, Z. W.; Zhan, D. Anal. Chem. 2013, 85, 1322-1326.

(20) Meyer, H.; Drewer, H.; Gründig, B.; Cammann, K.; Kakerow, R.; Manioli, Y.; Mokwa, W.; Rospert, M. Anal. Chem. 1995, 67, 11641170.

(21) Barker, A. L.; Unwin, P. R.; Gardner, J. W.; Rieley, H. Electrochem. Commun. 2004, 6, 91-97.

(22) Deiss, F.; Combellas, C.; Fretigny, C.; Sojic, N.; Kanoufi, F. Anal. Chem. 2010, 82, 5169-5175.

(23) Yasukawa, T.; Kaya, T.; Matsue, T. Anal. Chem. 1999, 71, 4637-4641.

(24) Nebel, M.; Neugebauer, S.; Eckhard, K.; Schuhmann, W. Electrochem. Commun. 2013, 27, 160-163.

(25) Alpuche-Aviles, M. A.; Wipf, D. O. Anal. Chem. 2001, 73, 48734881

(26) Gabrielli, C.; Huet, F.; Keddam, M.; Rousseau, P.; Vivier, V. J.

Phys. Chem. B 2004, 108, 11620-11626.

(27) Etienne, M.; Schulte, A.; Schuhmann, W. Electrochem. Commun. 2004, 6, 288-293.

(28) Macpherson, J. V.; Unwin, P. R. Anal. Chem. 2000, 72, 276285.

(29) Kranz, C.; Friedbacher, G.; Mizaikoff, B.; Lugstein, A.; Smolier, J.; Bertagnolli, E. Anal. Chem. 2001, 73, 2491-2500.

(30) Davoodi, A.; Pan, J.; Leygraf, C.; Norgren, S. Electrochim. Acta 2007, 52, 7697-7705.

(31) Pust, S. E.; Salomo, M.; Oesterschulze, E.; Wittstock, G. Nanotechnology 2010, 21, 105709/105701-105709/105712.

(32) Anne, A.; Chovin, A.; Demaille, C.; Lafouresse, M. Anal. Chem. 2011, 83, 7924-7932.

(33) Gullo, M. R.; Frederix, P. L. T. M.; Akiyama, T.; Engel, A.; de Rooij, N. F.; Staufer, U. Anal. Chem. 2006, 78, 5436-5442.

(34) Treutler, T. H.; Wittstock, G. Electrochim. Acta 2003, 48, 29232932.

(35) Comstock, D. J.; Elam, J. W.; Pellin, M. J.; Hersam, M. C. Anal. Chem. 2010, 82, 1270-1276.

(36) Wipf, D. O.; Bard, A. J. Anal. Chem. 1992, 64, 1362-1367.

(37) Edwards, M. A.; Whitworth, A. L.; Unwin, P. R. Anal. Chem.

2011, 83, 1977-1984.

(38) Ludwig, M.; Kranz, C.; Schuhmann, W.; Gaub, H. E. Rev. Sci. Instrum. 1995, 66, 2857-2860.

(39) James, P.; Garfias-Mesias, L. F.; Moyer, P. J.; Smyrl, W. H. J. Electrochem. Soc. 1998, 145, 64-66.

(40) Takahashi, Y.; Shevchuk, A. I.; Novak, P.; Babakinejad, B.; MacPherson, J.; Unwin, P. R.; Shiku, H.; Gorelik, J.; Klenerman, D.;
Korchev, Y. E.; Matsue, T. Proc. Natl. Acad. Sci. U.S.A. 2012, 109, 11540-11545.

(41) Cortes-Salazar, F.; Träuble, M.; Li, F.; Busnel, J.-M.; Gassner, A.L.; Hojeij, M.; Wittstock, G.; Girault, H. H. Anal. Chem. 2009, 81, 6889-6896.

(42) Cortes-Salazar, F.; Momotenko, D.; Lesch, A.; Wittstock, G.; Girault, H. H. Anal. Chem. 2010, 82, 10037-10044.

(43) Lesch, A.; Momotenko, D.; Cortes-Salazar, F.; Wirth, I.; Tefashe, U. M.; Meiners, F.; Vaske, B.; Girault, H. H.; Wittstock, G. J. Electroanal. Chem. 2012, 666, 52-61.

(44) Lesch, A.; Vaske, B.; Meiners, F.; Momotenko, D.; CortesSalazar, F.; Girault, H. H.; Wittstock, G. Angew. Chem., Int. Ed. 2012, 51, 10413-10416.

(45) Lesch, A.; Momotenko, D.; Cortés-Salazar, F.; Roelfs, F.; Girault, H. H.; Wittstock, G. Electrochim. Acta 2013, 110, 30-41.

(46) Nunes Kirchner, C.; Hallmeier, K. H.; Szargan, R.; Raschke, T.; Radehaus, C.; Wittstock, G. Electroanalysis 2007, 19, 1023-1031.

(47) Wittstock, G.; Asmus, T.; Wilhelm, T. Fresenius J. Anal. Chem. 2000, 367, 346-351.

(48) Fulian, Q.; Fisher, A. C.; Denuault, G. J. Phys. Chem. B 1999, 103, 4387-4392.

(49) Sklyar, O.; Wittstock, G. J. Phys. Chem. B 2002, 106, 74997508.

(50) Cornut, R.; Bhasin, A.; Lhenry, S.; Etienne, M.; Lefrou, C. Anal. Chem. 2011, 83, 9669-9675.

(51) Meyer, E.; Hug, H. J.; Bennewitz, R. Scanning Probe Microscopy: The Lab on a Tip; Springer: Berlin, Germany; 2004.

(52) Tefashe, U. M.; Wittstock, G. C. R. Chim. 2013, 16, 7-14. 